\title{
Mechanical analysis of the bending behaviour of plant stems
}

\author{
Tom Leblicq*, Simon Vanmaercke, Herman Ramon, Wouter Saeys \\ KU Leuven - University of Leuven, Department of Biosystems (BIOSYST), Division of \\ Mechatronics, Biostatistics and Sensors (MeBioS), Kasteelpark Arenberg 30, 3001 \\ Leuven, Belgium
}

1 Abstract

2 In order to optimise the processing of stem crops, insight into the deforma-

3 tion behaviour of the crop and the interaction between crop and machine is

4 essential. Most research in the area of mechanical and physical properties of

5 crop stems is focused on characterising the agricultural products to the point

6 of failure using mechanical parameters and empirical relations. No studies

have been conducted on the processes which lead to failure of stems and on

s the processes that take place after failure. In this paper it is shown that

9 the bending behaviour of wheat and barley stalks is very similar to that of

steel tubes. Two consecutive phases can be distinguished: ovalisation and buckling. During ovalisation the forces on the wall tend to flatten the crosssection. When this process continues the flexural stiffness is reduced until the structure becomes unstable and buckles. The cross-section locally completely flattens. This deformed cross-section offers virtually no resistance to bending. Mechanical models described in literature have successfully been adapted to describe the bending behaviour of crop stalks during both phases ( $R^{2}>0.98$ for ovalisation and $R^{2}>0.97$ for buckling). The crop species,

${ }^{*}$ Corresponding author.

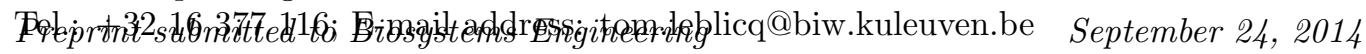


19 ence the bending process significantly. The presence of a core-rind structure 20 increases the bending resistance of the stems.

${ }_{21}$ Keywords: Stem crops, Ovalisation, Buckling, Core-rind structure 


\begin{tabular}{|ll|}
\hline $\begin{array}{l}\text { Nomenclature } \\
\theta\end{array}$ & Mechanism angle defined in figure $7 \mathrm{~b}(\mathrm{deg})$ \\
$\theta^{\prime}$ & Bending angle (deg) \\
$\theta_{y}$ & Yormalised bending angle (deg) \\
$\nu$ & Poisson's ratio of the stem (-) \\
$\nu_{c}$ & Poisson's ratio of the core (-) \\
$\xi$ & A dimensionless measure of the flattening at the extreme fibre $(-)$ \\
$\sigma_{y}$ & Yield stress (Pa) \\
$\phi_{0}, \phi_{0}^{\prime}$ & Mechanism angles defined in figure $5 \mathrm{c}(\mathrm{deg})$ \\
$a, b, c, k$ & Model parameters \\
$c$ & Core thickness (m) \\
$C$ & Curvature (-) \\
$D_{1}$ & Diameter of the flattened cross section (m ) \\
$E$ & Young's modulus of the stem (Pa) \\
$E_{c}$ & Young's modulus of the core (Pa) \\
$F_{y}$ & Force at buckling (N) \\
$H$ & Deformation (m) \\
$H_{y}$ & Deflection at buckling (m) \\
$I_{0}$ & Second moment of area for a circular cross section $\left(\mathrm{m}^{4}\right)$ \\
$I_{b}$ & Second moment of area of the deformed cross section $\left(\mathrm{m}^{4}\right)$ \\
$l$ & Size of the diamond shape (m) \\
$L$ & Support distance (m) \\
$L_{0}$ & Size of the buckling hinge (m) \\
\hline
\end{tabular}




\begin{tabular}{|ll|}
\hline$M_{y}$ & Moment at buckling $(\mathrm{N} \mathrm{m})$ \\
$r$ & Radius $(\mathrm{m})$ \\
$t$ & Wall thickness $(\mathrm{m})$ \\
$U_{1}$ & Energy for circumferential bending $(\mathrm{J})$ \\
$U_{2}$ & Energy for longitudinal stretching $(\mathrm{J})$ \\
\hline
\end{tabular}

\section{Introduction}

Biological stem crops such as wheat, barley, oats, rice and grasses are harvested, chopped and compressed in various agricultural machines. For optimisation of these processes, knowledge of both the deformation behaviour of the crop and of the interaction between crop and machine is required.

\subsection{Bulk models for crop compression}

Many researchers have investigated the bulk deformation of stem crops and the factors influencing this process. Several (empirical) models have been proposed for describing the bulk compression, including exponential (Faborode \& O’Callaghan, 1986; Ferrero et al., 1990; Nona et al., 2014), power law (Mewes, 1958) and polynomial forms (Sitkei, 1987). Faborode \& O'Callaghan (1986) and Nona et al. (2014) distinguished two consecutive phases during the compression of crops. The material changes from an initial two-phase mixture of solid particles and air into a predominantly solid form at high densities. In the first phase the stems rearrange and the air voids among stems are filled. It is hypothesised that friction plays the most important role during this phase. In the second phase the stems are compressed and thus flattened. 
For a more precise description, an additional phase can be added in between these two phases. In this phase, the stems are bent by contact with each other and by contact with machine components. The forces required for bending of stems are smaller than those required for compression. Due to bending the stems become more entangled and more air will be expelled. When a bulk of crop stalks is fed into an agricultural machine, the different phases take place. The bending phase often plays an important role in the total deformation of the crop because bending of crop stems already significantly takes place at lower forces, in contrast to compression.

\subsection{DEM simulations of crop processing}

The bulk deformation behaviour of crops is determined by the physical and mechanical properties of the individual crop stems. Due to large variations in the crop characteristics and due to complex interactions between the crop stems, the estimation of bulk deformation behaviour based on stem properties is nearly impossible. To the knowledge of the authors no research has been published on this subject. A bulk modelling approach is moreover unfit for describing the interactions between individual crop stems and machine components. Therefore, discrete element modelling (DEM) has been proposed as a better way to model the behaviour of particulate systems (Tijskens et al., 2003). Very recently DEM was used to create virtual crop stems (Lenaerts et al., 2014). Linear elastic models were used to describe the forces during deformation of the stems. These models resulted in realistic behaviour for relatively small forces and deformations. For larger deformations the deformations are, however, plastic. Annoussamy et al. (2000) showed that the bending behaviour of wheat stems is only linear for the initial deformations. 
More realistic models are required for obtaining accurate simulations. For this more insight is needed into the phenomena which occur during deformation of individual crop stalks.

\subsection{Deformation of individual crop stems}

Most research in the area of mechanical and physical properties of crop stems can be classified as the application of fundamental principles of mechanics to the mechanical behaviour of crop stems and the adaptation of test procedures to agricultural materials (Nazari Galedar et al., 2008). Methods and procedures for measuring physical and mechanical parameters of agricultural products have been described by Huisman (1978), Mohsenin (1986) and O'Dogherty et al. (1995).

Many studies have been conducted on characterising crop stems at the particle level to the point of failure. This was done for different crops and various conditions (Bright \& Kleis, 1964; O'Dogherty et al., 1995; Annoussamy et al., 2000; Yu et al., 2006; Nazari Galedar et al., 2008). Often an empirical relation is found between these mechanical parameters and the physical crop characteristics. O'Dogherty et al. (1995) found values for the tensile strength of wheat straw in the range from 21.2 MPa to 31.2 MPa. The shear strength was determined to range from 4.91 MPa to 7.26 MPa. Young's modulus ranged from 4.76 GPa to $6.58 \mathrm{GPa}$ and the rigidity modulus was in the range from $267 \mathrm{MPa}$ to $547 \mathrm{MPa}$. Yu et al. (2006) reported values for the ultimate tensile stress of switchgrass between 9.3 MPa and $213 \mathrm{MPa}$. The ultimate shear stress ranged from 6.9 MPa to 39.9 MPa. Nazari Galedar et al. (2008) determined the Young's modulus of alfalfa to range from 0.79 GPa to 3.99 GPa. From these studies, it is clear that large variations exist 
within one species.

Mechanical properties of crops are influenced by the moisture content and the plant maturity of the stems, the internode position, the soil type and the temperature. An increase in moisture content results in a decrease in maximum bending stress, tensile stress, torsional stress, Young's modulus and rigidity modulus, while the shear stress increases (Bright \& Kleis, 1964; Annoussamy et al., 2000; Nazari Galedar et al., 2008). However, when the plant ages, Young's modulus increases (Bright \& Kleis, 1964; O'Dogherty et al., 1995). As the diameter and cross-sectional area decrease towards the ear of the plant, the tensile and shear stresses at failure increase when moving in this direction. Moreover, due to the higher proportion of hemicelluloses, Young's modulus also increases (Annoussamy et al., 2000).

\subsection{Contributions of this study}

Few studies have been reported which aimed at unravelling the factors that influence the mechanical failure of plant stems. To the knowledge of the authors, no studies have been conducted on the processes which lead to failure of crop stems and on the processes that take place after failure. To illustrate the complexity of the deformation behaviour of plant stems threepoint bending tests were conducted. An experimental set-up was used where the stems were placed on two metal support. The pressing element had the same, circular, shape as a stem (figure 1). This geometry was chosen to simulate the interaction between different crop stems. The resistance to bending can be displayed as the measured force as a function of the deformation by the plunger or as the measured moment as a function of the applied angle. The force-deformation curve for three-point bending of wheat stems is pre- 


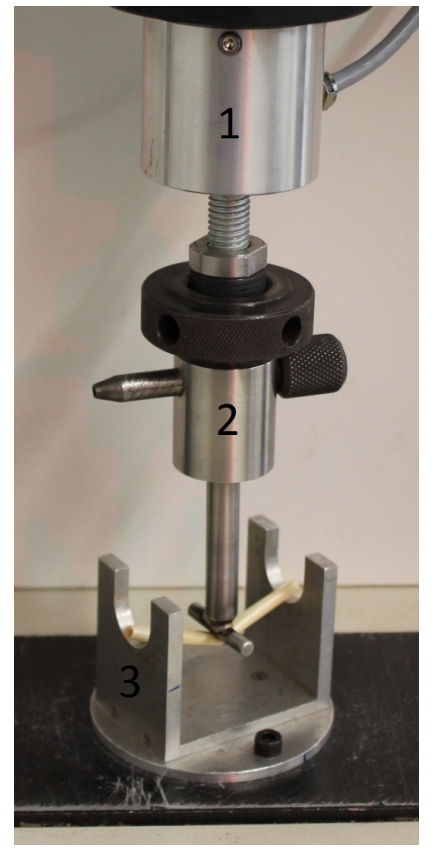

Figure 1: Measurement set-up for three-point bending of crop stems (1. load cell, 2. rod with pressing element and adopter, 3. supports)

sented in figure 2a. It can be seen that linearity is only an approximation for small deformations. For optimisation of crop processing machines using simulations (e.g. using DEM) more insight is needed into the phenomena which occur during interaction and deformation of crop stems.

The aim of this study was to model the processes which lead to failure of plant stems due to bending and to gain insight into the phenomena. The factors influencing these processes were also investigated. Since no reports were found describing the mechanical processes taking place during bending of plant stems, materials with similar bending behaviour were sought and the mechanical models describing deformation and failure were adapted. 


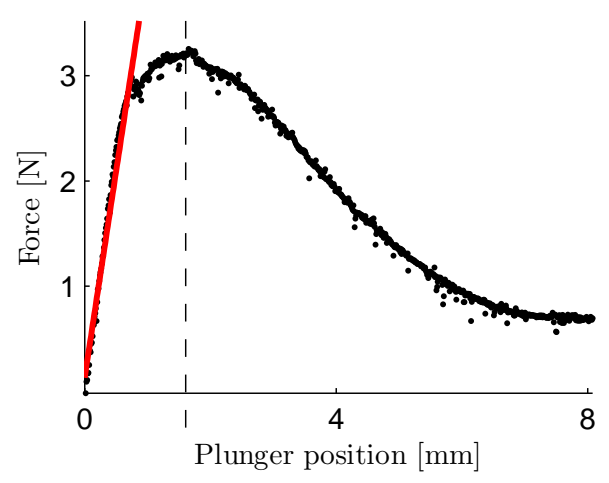

(a)

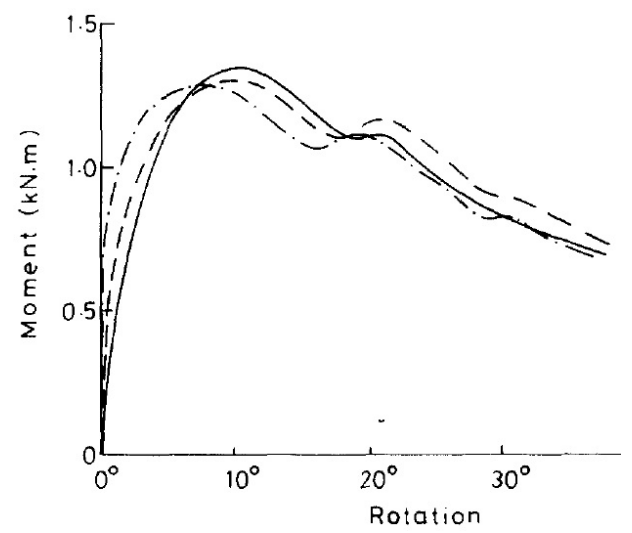

(b)

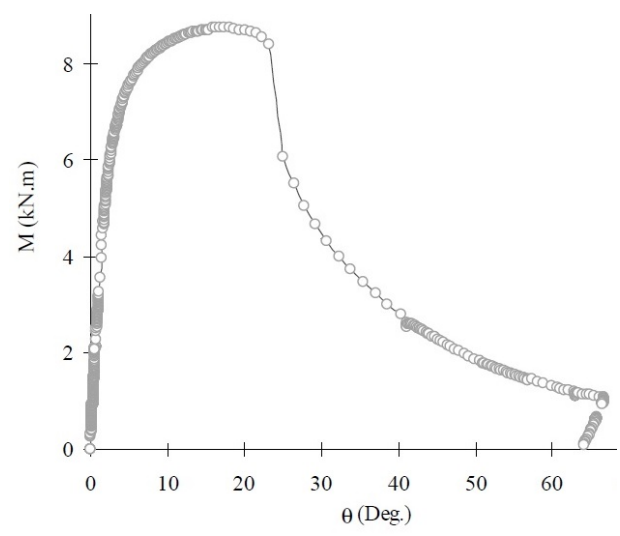

(c)

Figure 2: (a) Typical force-deformation curve for three point bending of wheat straw. A linear elastic model (equation (26)) was fitted to the first part of the data (red line). The dashed line indicates the end of ovalisation and the start of buckling. (b) Angle-moment curves for bending of hollow steel tubes (Mamalis et al., 1989). (c) Angle-moment curve for bending of hollow steel tubes (Elchalakani et al., 2002) 


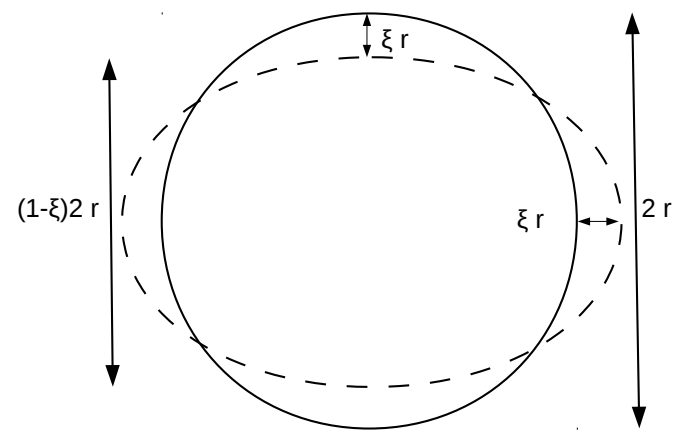

Figure 3: - undeformed cross-section, - - ovalised cross-section

\section{Theory}

A plant stem is essentially a long, thin-walled circular tube. It should, therefore, not come as a surprise that the observed force-deformation curve for wheat straw (figure 2a) corresponds to those described and measured for the bending of steel tubes (Mamalis et al. (1989), figure 2b; Elchalakani et al. (2002), figure 2c). Two consecutive phases are typically distinguished in the bending of tubes: ovalisation and buckling.

\subsection{Ovalisation}

When a tube is bent the inner side of the tube is longitudinally compressed while the outer side is stretched. Both this compression and tension result in a resistance of the tube against the bending moment. As both have a component directed towards the centre of the tube, the stresses cause a flattening of the circular cross-section into an oval shape (figure 3). This phase in bending is called ovalisation (Brazier, 1927).

The total strain energy $(U)$ for elastic deformation of a tube by ovalisation is the sum of the energy for circumferential bending (ovalisation, $U_{1}$ ) and 
longitudinal stretching (bending of the deformed tube, $U_{2}$ )(Calladine, 1989):

$$
\begin{gathered}
U=U_{1}+U_{2} \\
U_{1}=\frac{3}{8} \pi E t h^{2} \frac{\xi^{2}}{r}
\end{gathered}
$$

142 with:

$$
h=\frac{t}{\left(1-\nu^{2}\right)^{\frac{1}{2}}}
$$

143 144 145 $\nu$ is Poisson's ratio.

$$
U_{2}=\frac{1}{2} C^{2} E \pi I_{b}
$$

146 with:

$$
I_{b}=I_{0}\left(1-\frac{3}{2} \xi\right)
$$

147 where $C$ is the curvature of the deformed tube, $I_{b}$ is the second moment of 148 area of the deformed cross section and $I_{0}$ is the second moment of area for a 149 circular cross section $\left(I_{0}=\pi r^{3} t\right)$. The theorem of minimum strain energy:

$$
\frac{d U}{d \xi}=0
$$

150 is used to determine the ovalisation of the tube. This results in a flattening 151 of: 


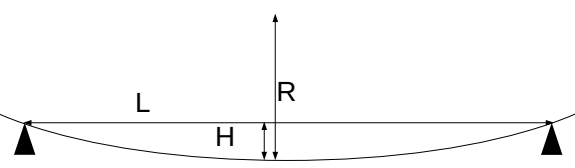

Figure 4: Relationship between curvature and deformation

$$
\xi=\frac{C^{2} r^{4}}{h^{2}}
$$

152 We can find an expression for the bending moment $(M)$ by means of the 153 relation (Calladine, 1989):

$$
M=\frac{d U}{d C}
$$

154 By combining (1), (2), (4) and (8), the bending moment can be calculated 155 as:

$$
M=\frac{\pi}{2} \operatorname{Er}^{3} t\left[2 C-\frac{3 r^{4} C^{3}\left(1-\nu^{2}\right)}{t^{2}}\right]
$$

${ }_{156}$ The curvature $(C)$ can be expressed as:

$$
C=\frac{1}{R}=\frac{8 H}{4 H^{2}+L^{2}}
$$

${ }_{157}$ where $R$ is the radius of curvature and $L$ is the support distance. The mean158 ing of these parameters is illustrated in figure 4. By replacing the curvature 159 by the deflection of the stem $(H)$, equation 9 can be can be rewritten as:

$$
F_{\text {ovalisation }}=\frac{E}{L} \pi r^{3} t\left[\frac{16 H}{4 H^{2}+L^{2}}-\frac{3 r^{4}(8 H)^{3}\left(1-\nu^{2}\right)}{t^{2}\left(4 H^{2}+L^{2}\right)^{3}}\right]
$$


Equation (11) describes the relationship between the bending force $(F)$ and lateral deformation $(H)$ up to the point of failure using five parameters: the support distance $(L)$, Young's modulus $(E)$, Poisson's ratio $(\nu)$, the stem radius $(r)$ and the wall thickness $(t)$. The point of failure is defined as the point of maximum force $\left(F_{y}\right)$. The corresponding lateral deformation is $H_{y}$. At the point of failure the transition between ovalisation and buckling takes place (figure 2a).

\subsection{Buckling}

During ovalisation the cross-section flattens due to the applied bending moment and the flexural stiffness decreases. Brazier (1927) showed that, under steadily increasing curvature, the bending moment reaches a maximum value. Under force-based deformation, the structure becomes unstable after this point of maximum bending moment has been passed. A kink is suddenly formed. This involves a complete local flattening of the cross-section, which offers virtually no resistance to bending (Calladine, 1989). This process is known as buckling. The point of buckling is not associated with an initial imperfection, but with the fact that bending of an initially straight tube is essentially non-linear in character. The effect of buckling on a wheat stem is illustrated in figures 5b, 6a and 7a. It can be seen that the collapse of a stem under bending involves the formation of plastic hinges. These plastic hinges are best described as local plastic mechanisms, because they involve large localised plastic deformation with geometrical folding (Elchalakani et al., 2002). The plastic deformations are distributed along the length of the stem and become more concentrated at the plastic hinge line. Measurements indicate that the flattened region increases as the bending increases (Elchalakani 
et al., 2002). Models for predicting the moment-rotation response of circular tubes subjected to bending were, among others, developed by Mamalis et al. (1989), Elchalakani et al. (2002) and Poonaya et al. (2009). They all divided the bending process into three phases. A linear elastic phase, a second phase with a constant bending moment (the ovalisation plateau) and the structural collapse in the third phase. The first two phases correspond to the ovalisation described by Brazier (1927). Although dividing ovalisation into two phases provided good results for steel tubes of large $D / t$-ratio (15 $<D / t<60)$, this description is less accurate for plant stems with smaller $D / t$-ratio $(6<D / t<10)$. The third phase can, however, be used to describe the buckling-phenomena.

Elchalakani et al. (2002) developed a closed-form solution for the momentrotation response during pure bending of circular hollow steel tubes for varying $D / t$. They described the energy dissipated due to buckling. A few assumptions were made:

- The deformations are inextensional, i.e. the circumference of the tube remains constant (figure 5).

- The buckling mechanism is assumed to articulate about a single central plastic hinge (figure 6).

- The hinge lines are assumed straight.

- A diamond shape is formed due to buckling (figure 6).

- Once the diamond shape is formed it remains constant.

- The deformation decreases with the distance from the hinge line. This relation is assumed linear (figure 7). 


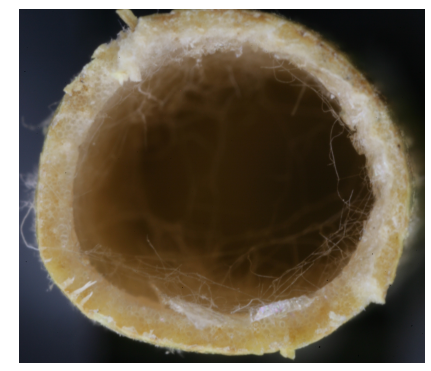

(a)

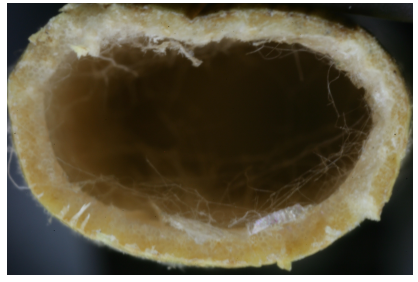

(b)

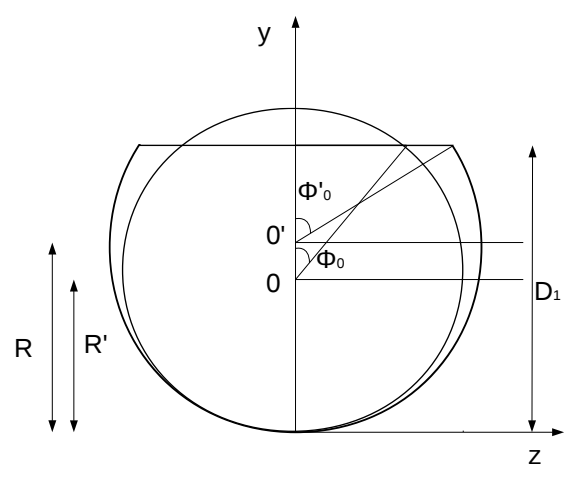

(c)

Figure 5: (a) Undeformed cross-section, (b) Flattened and ovalised cross-section, (c) Cross-section deformations

209 The internal work dissipated during crushing of the tube is composed of 210 four major components: the plastic deformation of the central hinge (12), 211 flattening of the diamond shape region (13), bending over the hinge line (14) 212 and flattening of the circular region (the continuation of ovalisation) (15).

$$
\begin{gathered}
W 1=2 \phi_{0} r m_{p}(\pi-2 \alpha) \\
W 2=2 L_{0} m_{p} \phi_{0}^{2} \\
W 3=4 m_{p} \phi_{0}^{\prime} \frac{l^{2}}{L_{0}}
\end{gathered}
$$




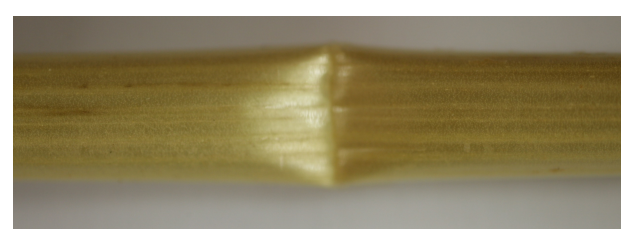

(a)

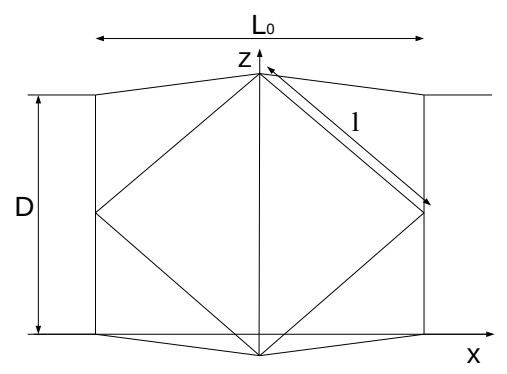

(b)

Figure 6: Deformation of a wheat stem by forming the diamond shape. (a) A wheat stem after buckling, (b) The diamond model

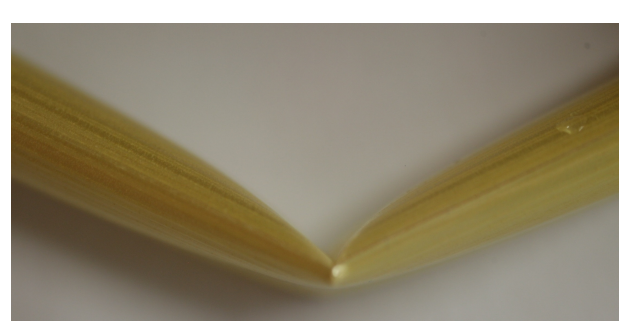

(a)

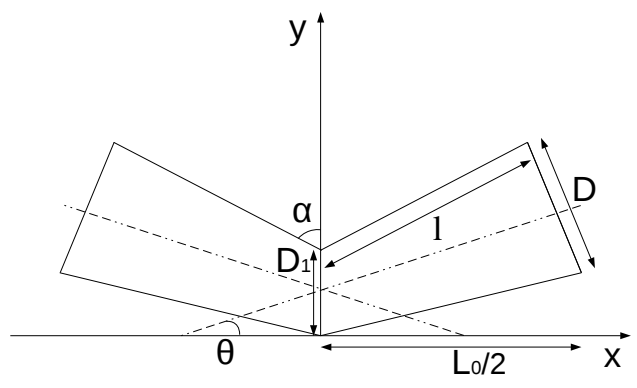

(b)

Figure 7: Longitudinal deformation of a wheat stem. (a) A wheat stem after buckling, (b) Longitudinal section deformations 


$$
W 4=2 L_{0} m_{p}\left(\pi-\phi_{0}\right)\left(\phi_{0}^{\prime}-\phi_{0}\right)
$$

213 with:

$$
m_{p}=\frac{\sigma_{y} t^{2}}{4}
$$

214

with:

$$
\phi_{0}^{\prime}=\cos ^{-1}\left(\frac{A_{1}^{2}-1}{A_{1}^{2}+1}\right)
$$

$$
A_{1}=2\left(\frac{c+k \theta^{\prime}}{a+b \theta^{\prime}}\right)
$$

${ }_{224}$ The components of the bending moment can be written using the following 225 equation:

$$
D_{1}=r^{\prime}\left(1+\cos \phi_{0}^{\prime}\right)
$$

and so: 


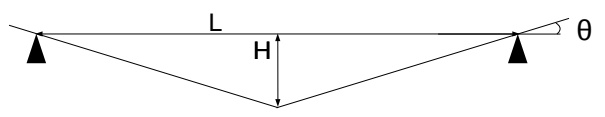

Figure 8: The relation between angle and deformation

$$
M_{i}=\frac{d}{d \theta^{\prime}} \frac{d \theta^{\prime}}{d \theta} W_{i}
$$

226

The total bending moment is the sum of the above components:

$$
M=M_{1}+M_{2}+M_{3}+M_{4}
$$

Equation 22 describes the total bending moment during buckling for an applied bending angle. The determination of these individual terms are given by Elchalakani et al. (2002). The detailed equation can be found in the appendix.

In this study the force-deformation behaviour of crop stems is studied. Therefore the moment-rotation equation of Elchalakani et al. (2002) (equation 22) is converted into its force-deformation equivalent. The total moment can be replaced by a force by taking the support distance into account $\left(F=M L^{-1}\right)$. The applied bending angle can then be described as a function of the deformation ( figure $8(H)$ ):

$$
\theta=\tan ^{-1}\left(\frac{2 H}{L}\right)
$$

${ }^{237}$ By substituting the yield stress $\left(\sigma_{y}\right)$ by the yield force $\left(F_{y}\right)$ (Timoshenko \& Gere, 1961)): 


$$
\sigma_{y}=\frac{\frac{L D}{2}}{\pi\left[\left(\frac{D}{2}\right)^{4}-\left(\frac{D}{2}-t\right)^{4}\right]} F_{y}
$$

by replacing the yield angle $\left(\theta_{y}\right)$ by the deflection at buckling $\left(H_{y}\right)$ (equation 23) and by substitution of $I_{0}, m_{p}$ (equation 16) and $A_{1}$ (equation 20) a model which describes the force as a function of the applied deformation can be obtained. Because of the many substitutions, the final equation is too large to be displayed here. The equation can, however, easily be obtained by doing the substitutions as described above. Equation 25 gives a summary of this equation.

$$
F_{\text {buckling }}=f\left(F_{y}, H_{y}, L, r, t, a, b, c, k, H\right)
$$

The model describes the forces during buckling and therefore starts were the ovalisation model (11) stopped. The model is a function of $F_{y}$ and $H_{y}$ (which describe the start of buckling and have the same value as the force and deformation at the end of ovalisation), of $L, r$ and $t$ (which have the same values as for ovalisation) and of the empirical parameters $a, b, c$ and $k$.

\section{Materials and methods}

To determine the validity of equations (11) and (25), 60 wheat stems (harvested in Mechterstädt, Germany during the summer of 2012), 60 wheat stems (harvested in Leuven, Belgium during the summer of 2013) and 60 barley stems (harvested in La Luisiana, Spain during the summer of 2013) were subjected to bending. The samples were collected randomly from the fields. Extra crop stems were collected for determination of the moisture 
content. This moisture content was measured by drying the samples in an oven at $103^{\circ} \mathrm{C}$ for 24 hours according to the ASABE Standard S358.2 (Standards ASABE, 2006). The moisture contents of the Spanish barley and the Belgian and German wheat were 8.9, 8.5 and 10.4\% (wb), respectively.

Prior to the measurements, petioles and leaves were removed and the stems were cut into pieces $60 \mathrm{~mm}$ long. The stems were placed on two metal supports $50 \mathrm{~mm}$ apart $(L)$ and then loaded midway with a metal plunger (figure 1). The plunger was rounded with a diameter similar to that of the diameter of the stems $(4 \mathrm{~mm})$. The plunger was driven at a constant speed by a universal testing system (UTS testsysteme GmbH, type UTS 5 K, Germany). The location of the plunger $(H)$ was recorded for each time step. Three loading rates were applied $\left(0.25 \mathrm{~mm} \mathrm{~s}^{-1}, 1 \mathrm{~mm} \mathrm{~s}^{-1}\right.$ and $\left.2.5 \mathrm{~mm} \mathrm{~s}^{-1}\right)$. For each loading rate 20 measurements were performed per location. The bending force $(F)$ was measured by a force transducer (Hottinger Baldwin Messtechnik GmbH, type U1A 10N, Germany). These experiments resulted in force-deformation profiles similar to the one displayed in figure $2 \mathrm{a}$.

The force-deformation profiles were used to determine the model parameters from equations (11) and (25) and to evaluate the validity of these models. Young's modulus was estimated for every crop stalk by least squares fitting the following expression for a supported beam (Nazari Galedar et al., 2008) to the linear part of the deformation profile:

$$
E=\frac{F_{b} L^{3}}{48 H I_{0}}
$$

The result of this fit is illustrated in figure 2a for a wheat stem. Poisson's 
ratio for all stalks was fixed at a constant value of 0.3 in accordance with O'Dogherty (1989) who found values between 0.1 and 0.5 and Sitkei (1987) who reported values between 0.25 and 0.4 . The radius $(r)$ and the wall thickness $(t)$ of the stems were estimated through least squares fitting of equation (11) to the same force-deformation profiles (up to the point of failure). The support distance $(L)$, the stem radius $(r)$ and the wall thickness $(t)$ were given the same values for buckling as for ovalisation. Using these values the empirical parameters of the buckling model $(a, b, c$ and $k$ ) were estimated through least squares fitting of equation (25) to the second part of the force-deformation profile.

\section{Results and discussion}

To evaluate the ovalisation and buckling model, both were fitted to the measured data and the estimated model parameters were compared to measured crop parameters and to parameters described in literature.

\subsection{Ovalisation}

The least squares fitting of equation (11) to the force-deformation profile measured for a wheat stem is illustrated in figure 9 . It can be seen that the model describes the slower increase in force during ovalisation very well. The model fits the data for all measurements well up until the point of failure. The measured stem properties and estimated model parameters are summarised in table 2. It can be seen that the $R^{2}$ values for the least squares fits are high for all three locations and all loading rates. The estimated values for Young's modulus are in accordance with those described in literature (see section 1). 


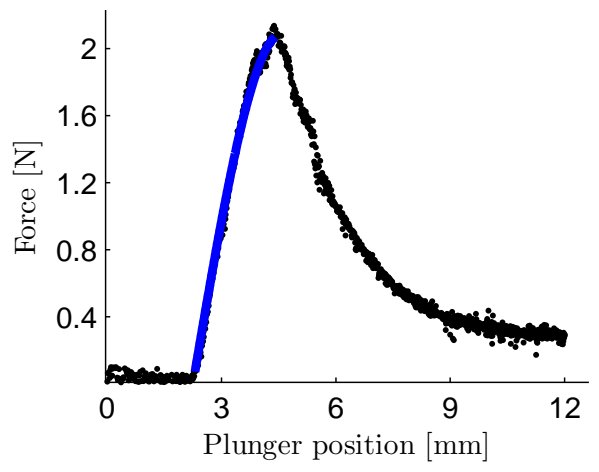

Figure 9: The model for ovalisation (11) fitted to the measured data (data from Mechterstädt at a loading rate of $1 \mathrm{~mm} \mathrm{~s}^{-1}$ )

Table 2: Crop characteristics (mean $(\mu)$ and standard deviation $(\sigma)$ ) $F_{y}=$ force at buckling, $H_{y}=$ deflection at buckling, $r=$ stem radius, $t=$ stem wall thickness, $E=$ Young's modulus $(m)=$ measured, $(e)=$ estimated by least squares fitting

\begin{tabular}{lccccccc}
\hline & & \multicolumn{2}{c}{ Germany (Wheat) } & \multicolumn{2}{c}{ Belgium (Wheat) } & \multicolumn{2}{c}{ Spain(Barley) } \\
\hline $\mathrm{N}$ & \multicolumn{3}{c}{60} & \multicolumn{2}{c}{60} & \multicolumn{2}{c}{60} \\
\hline & & $\mu$ & $\sigma$ & $\mu$ & $\sigma$ & $\mu$ & $\sigma$ \\
$F_{y}^{(m)}$ & {$[\mathrm{N}]$} & 3.12 & 1.43 & 4.42 & 1.59 & 6.67 & 2.26 \\
$H_{y}^{(m)}$ & {$[\mathrm{mm}]$} & 1.94 & 0.53 & 1.45 & 0.32 & 1.90 & 0.29 \\
$r^{(e)}$ & {$[\mathrm{mm}]$} & 1.19 & 0.17 & 1.93 & 0.32 & 1.56 & 0.25 \\
$t^{(e)}$ & {$[\mathrm{mm}]$} & 0.39 & 0.11 & 0.37 & 0.061 & 0.36 & 0.048 \\
$E^{(e)}$ & {$[\mathrm{GPa}]$} & 3.59 & 1.28 & 1.94 & 0.93 & 3.58 & 1.39 \\
$R^{2}$ & & 0.98 & 0.001 & 0.98 & 0.03 & 0.98 & 0.001 \\
\hline
\end{tabular}


As an extra validation of equation (11), the estimated stem radii and wall thicknesses were compared to the real values. The diameter was determined using a digital calliper (Mitutoyo, Japan). Most specimens were slightly elliptical in cross-section. For this reason the diameter was measured at the major and minor axis of the cross-section. However, equation (11) is based on an initial circular cross section. The mean of the measured diameters was therefore used as an equivalent diameter. The wall thickness was measured at the middle of the stem, where the force was applied, using a digital micrometer (Mitutoyo, Japan). For this reason the wall thickness was determined after bending. It was observed that for multiple measurements of the wall thickness, the values varied widely from one sample to another. Besides the biological variability, this is also due to the curvature of the stem which makes the measurements less accurate. The wall thickness was measured three times per sample and the average values were used.

The results of comparing the measured and estimated stem radii and wall thicknesses are shown in figures 10 and 11. In the ideal case, the measured and the estimated parameter values would be equal. In this case all points would lie on the bisector. It is clear that large variations exist between stems, even for stems from one species collected from the same field. A good correlation was found for the stem diameters $\left(R^{2}=0.84\right)$. The correlation for wall thickness was lower $\left(R^{2}=0.69\right)$. This can most likely be attributed to a larger measurement error. The slope of the regression line between the estimated and measured wall thicknesses was 0.73 , this is considerably lower than 1. This means that the higher wall thicknesses are underestimated. A closer look at figure 11 shows that this is especially the case for the wheat 


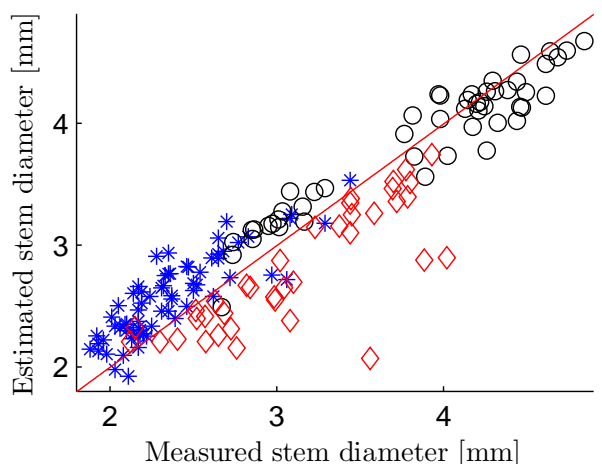

Figure 10: Measured diameter versus estimated diameter $\left(y=0.9985 x\left(R^{2}=0.84\right)\right)$ Germany $(*)$, Spain $(\diamond)$ and Belgium (o)

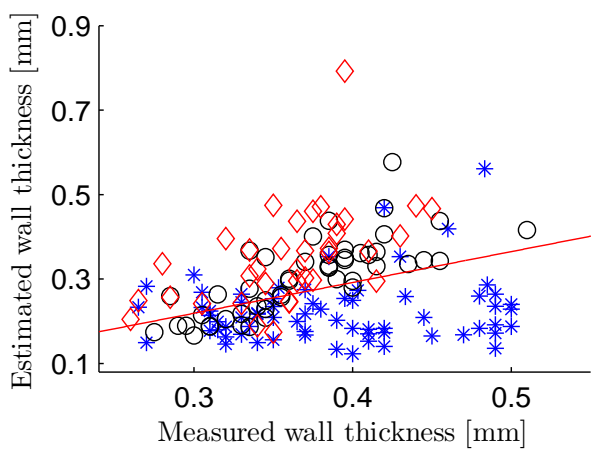

Figure 11: Measured wall thicknesses versus estimated wall thicknesses

$$
\left(y=0.7298 x\left(R^{2}=0.69\right)\right)
$$

Germany $(*)$, Spain $(\diamond)$ and Belgium $(\circ)$ 
stems collected in Mechterstädt, Germany. These differences between the measured and estimated values can be partially explained by two reasons. Primarily, the diameters of the stems from Germany were smaller than those from Spain and Belgium. As a result, the curvature is larger and the measurement of the wall thickness is thus less accurate. Secondly, the presence of a core-rind structure in many of the wheat stems made it difficult to determine the exact wall thickness. Although stems were chosen where the core had already died and largely disappeared, small amounts still remained. This could also explain the relatively large variation in measured wall thicknesses. When only the data from Spain and Belgium were used, a slope of 0.9321 and an average $R^{2}$-value of 0.89 were obtained. Therefore, it can be concluded that equation (11) is suitable for describing the processes which occur during the ovalisation phase of bending.

For each location three loading rates were applied. However, no significant effect ( $\mathrm{P}>0.05$ ) could be observed for the bending stiffness (Young's modulus) or the force and deformation at the point of failure. Few reports have been found on the effect of loading rate on the bending characteristics of individual stems. Moreover, the reports by various researchers are in disagreement. Tavakoli et al. (2009) and El-Hag et al. (1971) observed a significant effect of the loading rate on the Young's modulus of barley and cotton stalks. Zareiforoush et al. (2010), however, found no significant effect for rice stems. A significant effect of the loading rate on the bending strength was observed for the internode closest to the ear, but not for the other internodes (Tavakoli et al., 2009; Zareiforoush et al., 2010). The loading rate in these studies varied 
between $5 \mathrm{~mm} \mathrm{~min}^{-1}$ and $15 \mathrm{~mm} \mathrm{~min}^{-1}$.

For bulk compression tests, the effect of loading rate has been studied more extensively. Mohsenin (1986), Sitkei (1987) and Kaliyan \& Morey (2009) developed rheological models for predicting the mechanical behaviour of biological materials. They found that this behaviour depends on the stress, strain, strain rate and size and shape of the biological materials. The loading rates for bulk compression in those studies were, however, significantly higher than those in this study. Therefore, it is hypothesised that the loading rate also has an effect on the level of individual stems, but that it can only be observed at sufficiently high strain rates. This is however beyond the aims of this study and therefore it should be further investigated in future research.

The ovalisation phase ends at the maximum force $\left(F_{y}\right)$. At this point the deformation is $H_{y}$. Knowing and being able to predict these parameters is important because they indicate the start of buckling. Annoussamy et al. (2000) found that $F_{y}$ is dependent on the density, the mass per unit length and the moisture content of the stems. Brazier (1927) showed that the bending moment at which buckling takes place is independent of any parameter defining the tube. In theory, a tube buckles at an ovalisation of $\xi=2 / 9$. However, Calladine (1989) re-analysed the problem and found that the moment required for buckling is always lower than the Brazier moment. In figure 12 the correlation between the stem diameters $(D)$ and the maximum forces $\left(F_{y}\right)$ is plotted. The relation between the two parameters is calculated for every location. As both the diameters and the maximum forces are significantly different $(\mathrm{P}>0.05)$ for each location, it is clear that the relation is 


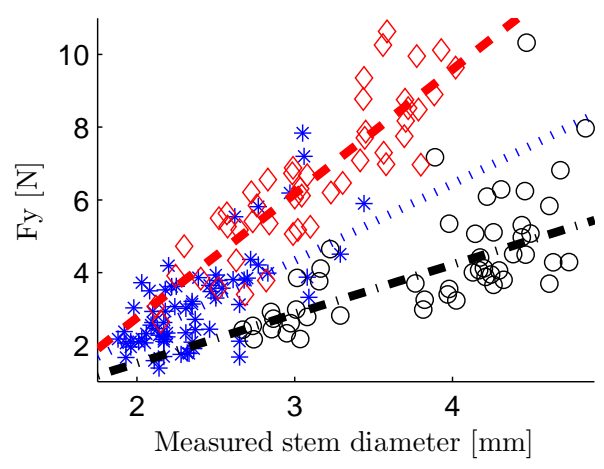

Figure 12: Stem diameter $(D)$ versus maximum force $\left(F_{y}\right)$

$$
\begin{gathered}
\text { Germany }(*) F_{y}=2114 D-2.02\left(R^{2}=0.60\right) \\
\text { Spain }(\diamond) F_{y}=1355 D-1.20\left(R^{2}=0.65\right) \\
\text { Belgium }(\circ) F_{y}=3415 D-4.07\left(R^{2}=0.79\right)
\end{gathered}
$$

different for the different locations. For $H_{y}$, however, the correlation with the physical parameters is less good. For all locations $R^{2}$ values below 0.35 were found for the correlation with diameter.

It should be noted that a sample length of $60 \mathrm{~mm}$ is rather short for a support distance of $50 \mathrm{~mm}$. During cutting of the samples, the adjacent portion of the stems is damaged and weakened. It is this portion that is supported. A longer sample length would ensure the absence of end effects.

\subsection{Buckling}

The result of fitting the buckling model (25) to the data is illustrated in figure 13 for a wheat stem from Mechterstädt. The model is suitable for describing the strong decrease in bending resistance due to flattening of the cross section. The average estimated model parameters and the corresponding standard deviations are summarised in Table 3 together with the 


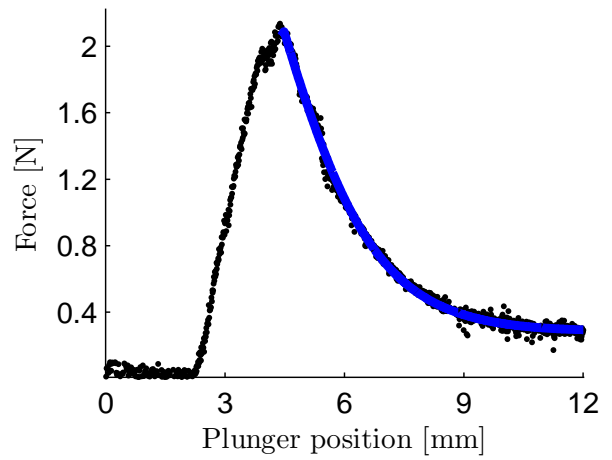

Figure 13: The model for buckling fitted to the measured data (data from Mechterstädt at a loading rate of $1 \mathrm{~mm} \mathrm{~s}^{-1}$ )

$R^{2}$ values. The order of magnitude of the parameters $(a, b, c$ and $k)$ is the same for all three locations. Since the parameters $a, b, c$ and $k$ are empirical parameters and, to the knowledge of the authors, no studies have been conducted on describing the buckling-behaviour of plant stems, the values of these parameters could not be compared to previously estimated ones. However, Elchalakani et al. (2002) found similar values for the empirical model parameters for steel tubes.

Both the physical crop parameters and the empirical model parameters are highly correlated. As a result, it is not possible to determine unaliased relations between both groups of parameters. To gain insight into the effect of the different parameters on the buckling process, a sensitivity analysis was conducted (figure 14). As a starting value the estimated model parameters for the wheat stem in figure 13 were used. Each parameter was then varied while the remaining parameters were kept constant.

An increase in the stem diameter has an effect on the rate at which 
the force drops due to buckling. A larger diameter results in a faster force decrease. The reverse is true for the wall thickness. As the wall thickness increases the energy required for buckling increases. The rate at which the force drops increases for an increase in the parameters $a, b$ and $c$. Buckling parameter $k$ not only has an effect on the force reduction rate but also on the deformation at the maximum force. This is in agreement with the findings of Elchalakani et al. (2002). In particular the parameters $b$ and $k$ have a significant effect on the buckling behaviour because they describe the rate of deformation as a function of the distance to the hinge line.

Table 3: Buckling parameters (mean $(\mu)$ and standard deviation $(\sigma)$ )

$$
a, b, c, k=\text { buckling parameters }
$$

\begin{tabular}{lcccccc}
\hline & Germany (Wheat) & \multicolumn{2}{c}{ Belgium (Wheat) } & \multicolumn{2}{c}{ Spain(Barley) } \\
\hline $\mathrm{N}$ & \multicolumn{2}{c}{60} & \multicolumn{2}{c}{60} & \multicolumn{2}{c}{60} \\
\hline & $\mu$ & $\sigma$ & $\mu$ & $\sigma$ & $\mu$ & $\sigma$ \\
$\mathrm{a}$ & 0.2177 & 0.3531 & 0.9732 & 0.3540 & 0.3365 & 0.1942 \\
$\mathrm{~b}$ & 0.0427 & 0.0405 & 0.1241 & 0.05538 & 0.1055 & 0.0448 \\
$\mathrm{c}$ & 0.1213 & 0.4048 & -0.8289 & 0.4153 & -0.5197 & 0.3250 \\
$\mathrm{k}$ & 0.3138 & 0.7346 & 0.6614 & 0.3385 & 0.4716 & 0.2448 \\
$R^{2}$ & 0.9877 & 0.0163 & 0.9796 & 0.0254 & 0.9901 & 0.0121 \\
\hline
\end{tabular}

\subsection{Core-rind structure}

Next to an outer shell of almost fully dense material, most plants also have a cellular core of lower density. Biologists refer to this as a "corerind" structure. This core is made up of soft, mostly white or slightly yellow coloured, parenchyma tissue (figure 15). In this study, the core-rind structure was only found in the wheat stems sampled in Mechterstädt, Germany. In 


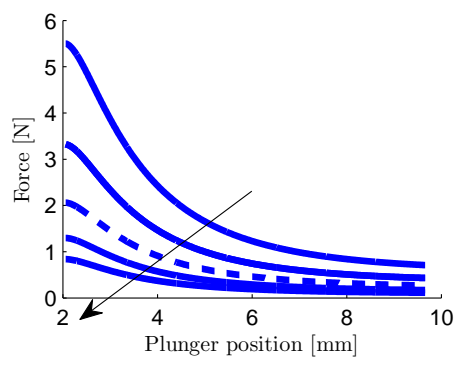

(a)

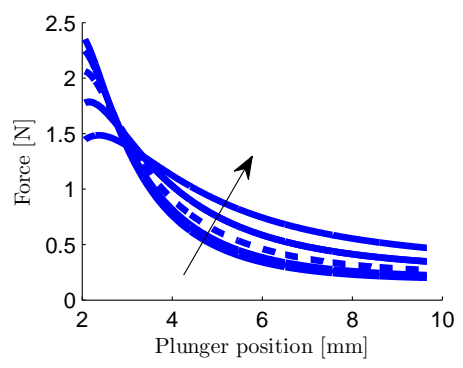

(c)

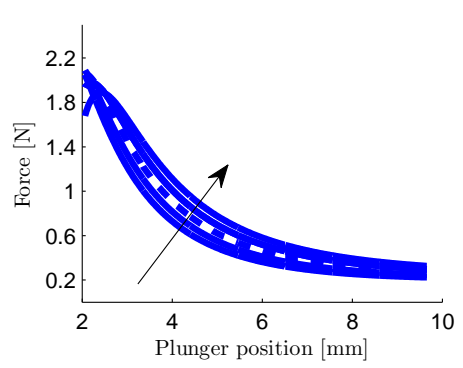

(e)

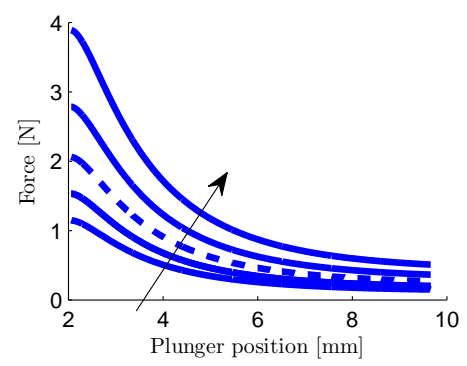

(b)

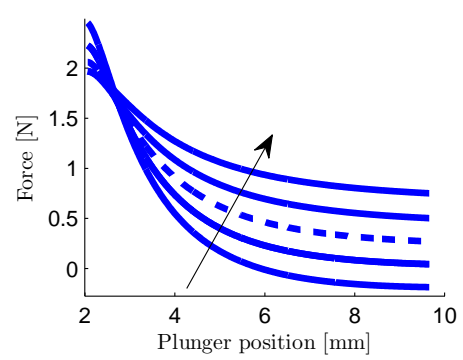

(d)

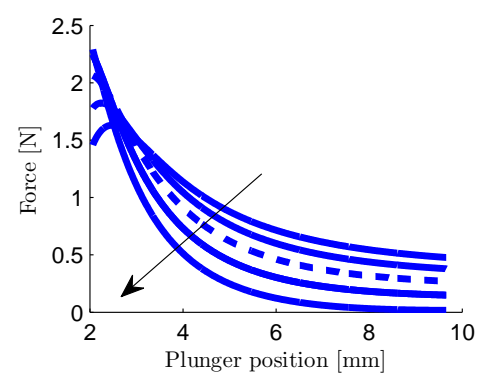

(f)

Figure 14: sensitivity analysis. The influence of the model parameters is displayed while all other parameters are kept constant. The arrow is oriented in the direction of an increase of the parameter. (a) Influence of $D: 0.6,0.8,1,1.2$ and 1.4 times the initial value (b) Influence of $t: 0.6,0.8,1,1.2$ and 1.4 times the initial value (c) Influence of $a$ : $0.25,0.5,1,2$ and 4 times the initial value (d) Influence of $b: 0.6,0.8,1,1.2$ and 1.4 times the initial value (e) Influence of $c: 0.8,0.9,1,1.1$ and 1.2 times the initial value (f) Influence of $k: 0.8,0.9,1,1.1$ and 1.2 times the initial value - - initial value: $D=0.00193 \mathrm{~m}, t=0.00041 \mathrm{~m}, F_{y}=2.0597 \mathrm{~N}, H_{y}=0.002061 \mathrm{~m}$,

$$
a=0.00377, b=0.01433,30=-0.010221, k=0.01436
$$

- multiples of the initial parameter values 

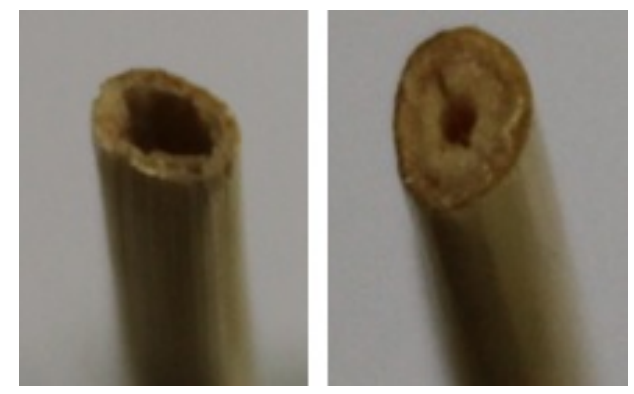

Figure 15: Cross sections of wheat stems (left: without a core structure, right: with core)

approximately fifty percent of the stems a core was present. No relation could be found between the presence of a core and other physical parameters.

The effect of a core has not yet been described for the bending behaviour of crop stems. For axial compression of metal and silicone rubber tubes it has, however, been shown that if an elastic core is sufficiently deep, it can carry additional load after buckling (Brush \& Almroth, 1962; Karam \& Gibson, 1995). The effect of the core on the force-deformation profile is presented in figure 16a. After the initial ovalisation, which also occurs without core, buckling phenomena occur. However, the force-deformation profile does not drop, but stays constant for a small amount of extra deformation. The stems then deform with reduced slope. The core offers extra resistance to bending. At higher forces the total resistance is insufficient and the force eventually drops rapidly.

To evaluate the effect of a core-rind structure on the bending characteristics of straw, 60 straw stems with core were subjected to three point bending. When the maximum force measured for the stems without core ( $N=60, \mu=3.12 N$ and $\sigma=1.30 N$ : see table 2) is compared to the force 


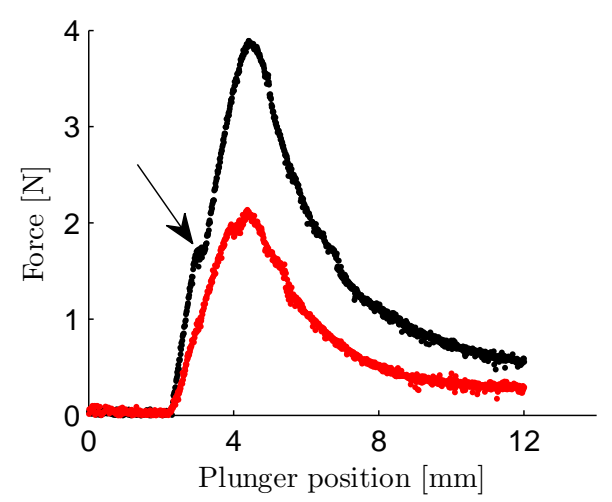

(a)

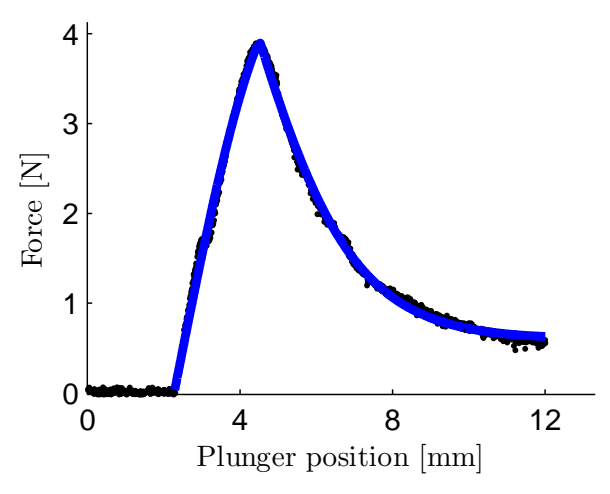

(b)

Figure 16: (a) Black: a typical force-deformation curve for three point bending of wheat straw with core-rind structure. (The arrow indicates the start of buckling of the outer shell). Red: wheat straw without core-rind structure. (b) Ovalisation (11) and buckling model (25) fitted to the data with core-rind structure.

at the start of buckling for the stems with core $(N=60, \mu=3.36 N$ and $\sigma=1.22 N)$ no significant difference can be observed at a significance level of $\alpha=0.05$. A significant difference is, however, observed when comparing the maximum force with $(N=60, \mu=4.86 N$ and $\sigma=1.43 N)$ and without core. The core contributes thus in a significant way to the strength and the bending resistance of wheat stems after buckling.

In figure 16b the ovalisation and buckling model are fitted to the data of a stem with core. The models fit the data well. An average $R^{2}$-value of 0.969 with a standard deviation of 0.0476 could be obtained for ovalisation and an average $R^{2}$-value of 0.956 with a standard deviation of 0.0563 was found for buckling. However, the estimated diameters and wall thicknesses were respectively a factor 1.23 and 2.40 larger than the measured values. This indicates that the presence of core-rind increases the effective bending 
resistance of the stems.

\section{Conclusions}

When bending a crop stem, two consecutive phases take place: ovalisation and buckling. The bending stresses that occur during ovalisation cause a flattening of the initial circular cross-section into an oval shape. An elastic energy approach, based on the work by Brazier (1927) and Calladine (1989), was used to model the force-deformation behaviour of crop stalks during ovalisation. The model was fitted to data from bending tests conducted on three crops. The model described the slower increase in force very well for all measurements $\left(R^{2}>0.98\right)$ up until the point of failure. The estimated stem diameters and wall thicknesses agreed well with the measured values.

The ovalisation of the cross-section continues up to the point when the bending moment reaches its maximum value and a kink is suddenly formed. The cross-section locally completely flattens, the bending resistance decreases and the buckling phase starts. A model developed by Elchalakani et al. (2002) was used to describe the strong decrease in bending resistance due to buckling. The model fitted well on the bending test data $\left(R^{2}>0.97\right)$. A sensitivity analysis showed that an increase in the stem diameter, the wall thickness and the buckling parameters $a, b$ and $c$ each cause an increase in the force reduction rate. The opposite is true for the parameter $k$. It was also shown that a cellular core of lower density than the outer shell can significantly increase the bending resistance of crop stems. 
475

476 477

478

$$
\begin{gathered}
M_{1}=\frac{m_{p}}{M_{y}} \frac{r}{L_{0}} E I_{0}\left(2 b\left[\pi-2 \sin ^{-1}(1-2 \sin \theta / 2)\right]+\frac{4 \theta_{y}\left(a+b \theta^{\prime}\right) \cos \theta / 2}{\sqrt{1-(1-2 \sin \theta / 2)^{2}}}\right) \\
M_{2}=\frac{m_{p}}{M_{y}} E I_{0}\left(4 b\left(a+b \phi^{\prime}\right)\right) \\
M_{3}=\frac{m_{p}}{M_{y}} \frac{r}{L_{0}} E I_{0}\left(\left(-\frac{4 F\left(L_{0}^{2} / 4+r^{2}\left(a+b \theta^{\prime}\right)^{2}\right)}{r L_{0}}\right)+\frac{1}{L_{0}}\left(8 r b\left(a+b \theta^{\prime}\right) \cos ^{-1} \frac{A_{1}^{2}-1}{1+A_{1}^{2}}\right)\right) \\
M_{4}=\frac{m_{p}}{M_{y}} E I_{0}\left(2\left(\pi-a-b \theta^{\prime}\right)(b+F)+2 b\left(-a-b \theta^{\prime}+\cos ^{-1} \frac{A_{1}^{2}-1}{1+A_{1}^{2}}\right)\right)
\end{gathered}
$$

480 with

$$
F=\left(\frac{\frac{\left(\frac{-2 b A_{1}^{2}}{a+b \theta^{\prime}}+\frac{2 k A_{1}}{c+k \theta^{\prime}}\left(A_{1}^{2}-1\right)\right)}{\left(1+A_{1}^{2}\right)^{2}}+\frac{\left(\frac{-2 b A_{1}^{2}}{a+b \theta^{\prime}}+\frac{2 k A_{1}}{c+k \theta^{\prime}}\right)}{1+A_{1}^{2}}}{\sqrt{1-\frac{\left(A_{1}^{2}-1\right)^{2}}{\left(1+A_{1}^{2}\right)^{2}}}}\right)
$$




\section{References}

Annoussamy, M., Richard, G., Recous, S., \& Gurif, J. (2000). Change in mechanical properties of wheat straw due to decompositon and moisture. Applied Engineering in Agriculture, 16, 657-664.

Brazier, L. G. (1927). On the flexure of thin cylindrical shells and other "thin" sections. Proceedings of the Royal Society of London, (pp. 104-116).

Bright, R., \& Kleis, R. (1964). Mass shear strength of haylage. Transactions of the ASAE, r, 100-101.

Brush, D., \& Almroth, B. (1962). Buckling of core-stabilized cylinders under axisymmetric external loads. Journal of Aerospace Science, 29, 1164-1170.

Calladine, C. R. (1989). Theory of shell structures. Cambridge University Press.

El-Hag, H., Kunze, O., \& Wilkes, L. (1971). Influence of moisture, drymatter density and rate of loading on ultimate strength of cotton stalks. Transactions of the ASAE, 4(2), 713-716.

Elchalakani, M., Zhao, X. L., \& Grzebieta, R. H. (2002). Plastic mechanism analysis of circular tubes under pure bending. International Journal of Mechanical Sciences, 44, 1117-1143.

Faborode, M. O., \& O'Callaghan, J. R. (1986). Theoretical analysis of the compression of fibrous agricultural materials. Journal of Agricultural Engineering Research, 35, 175-191. 
Ferrero, A., Horabik, J., \& Molenda, M. (1990). Density-pressure relationships in compaction of straw. Canadian Agricultural Engineering, 33, $107-111$.

Huisman, W. (1978). Moisture content, coefficient of friction and modulus of elasticity of straw in relation to walker losses in a combine harvester. American Society of Agricultural Engineers.

Kaliyan, N., \& Morey, R. V. (2009). Constitutive model for densification of corn stover and switchgrass. Biosystems Engineering, 104, 47-63.

Karam, G., \& Gibson, L. (1995). Elastic buckling of cylindrical shells with elastic coresi. analysis. International journal of solids and structures, 32, $1259-1283$.

Lenaerts, B., Aertsen, T., Tijskens, E., De Ketelaere, B., Ramon, H., De Baerdemaeker, J., \& Saeys, W. (2014). Simulation of grain-straw separation by discrete element modeling with bendable straw particles. Computers and Electronics in Agriculture, 101, 24-33.

Mamalis, A., Manolakos, D., Baldoukas, A., \& Viegelahn, G. (1989). Deformation characteristics of crashworthy thin-walled steel tubes subjected to bending. Proceedings of the Institution of Mechanical Engineers, Part $C$ : Journal of Mechanical Engineering Science, 203, 411-417.

Mewes, S. (1958). Zum verhalten von pressgutern in prestopfen (on the behaviour of compressed matter in pressure chambers). Landtechnische Forschung, 8, $154-164$. 
Mohsenin, N. N. (1986). Physical properties of plant and animial materials. Gordon and Breach, Science Publishers, Inc.

Nazari Galedar, M., Jafari, A., Mohtasebi, S., Tabatabaeefar, A., Sharifi, A., O’Dogherty, M., Rafiee, S., \& Richard, G. (2008). Effects of moisture content and level in the crop on the engineering properties of alfalfa stems. Biosystems Engineering, 101, 199-208.

Nona, K., Lenaerts, B., Kayacan, E., \& Saeys, W. (2014). Bulk compression characteristics of straw and hay. Biosystems Engineering, 118, 194-202.

O'Dogherty, M. (1989). A review of the mechanical behaviour of straw when compressed to high densities. Journal of Agricultural Engineering Research, 44, 241-265.

O’Dogherty, M. J., Huber, J. A., Dyson, J., \& Marshall, C. J. (1995). A study of the physical and mechanical properties of wheat straw. Journal of Agricultural Engineering Research, 62, 133-142.

Poonaya, S., Teeboonma, U., \& Thinvongpituk, C. (2009). Plastic collapse analysis of thin-walled circular tubes subjected to bending. Thin-walled structures, 47, 637-645.

Sitkei, G. (1987). Mechanics of agricultural materials. Elsevier Science Publishers.

Standards ASABE (2006). S358.2: Moisture measurement - Forages. St. Joseph, MI: American Society of Agricultural and Biological Engineers (ASABE). 
546 Tavakoli, H., Mohtasebi, S., \& Jafari, A. (2009). Effects of moisture content, 547 internode position and loading rate on the bending characteristics of barley ${ }_{548}$ straw. Journal of Agricultural Engineering Research, 55, 45-51.

549 Tijskens, E., Ramon, H., \& De Baerdemaeker, J. (2003). Discrete element modelling for process simulation in agriculture. Journal of sound and vibration, 266, 493-514.

Timoshenko, S., \& Gere, J. M. (1961). Theory of elastic stability. McGrawHill Book Company, Inc.

Yu, M., Womac, A., Igathinathane, C., Ayers, P., \& Buschermohle, M. (2006). Switchgrass ultimate stresses at typical biomass conditions available for processing. Biomass and Bioenergy, 30, 214-219.

Zareiforoush, H., Mohtasebi, S., Tavakoli, H., \& Alizadeh, M. (2010). Effect of loading rate on mechanical properties of rice (Oryza sativa L.) straw. Australian Journal of Crop Science, 4, 190-195. 